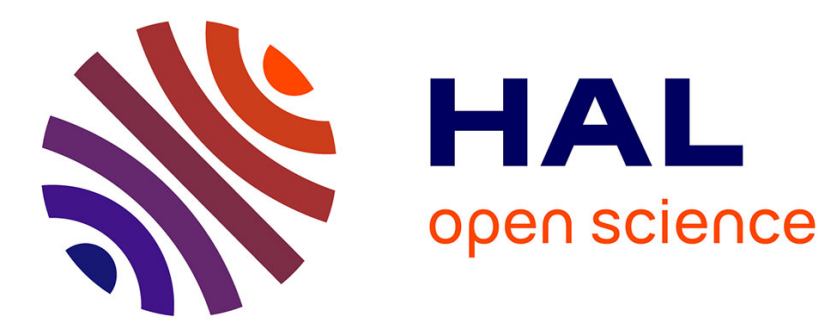

\title{
Towards the Automatic Tuning of Linear Controllers Using Iterative Learning Control Under Repeating Disturbances
}

Oktay Koçan, Charles Poussot-Vassal, Augustin Manecy

\section{- To cite this version:}

Oktay Koçan, Charles Poussot-Vassal, Augustin Manecy. Towards the Automatic Tuning of Linear Controllers Using Iterative Learning Control Under Repeating Disturbances. ECC 2019, Jun 2019, NAPLES, Italy. 10.23919/ECC.2019.8796268 • hal-02902578

\section{HAL Id: hal-02902578 \\ https://hal.science/hal-02902578}

Submitted on $20 \mathrm{Jul} 2020$

HAL is a multi-disciplinary open access archive for the deposit and dissemination of scientific research documents, whether they are published or not. The documents may come from teaching and research institutions in France or abroad, or from public or private research centers.
L'archive ouverte pluridisciplinaire HAL, est destinée au dépôt et à la diffusion de documents scientifiques de niveau recherche, publiés ou non, émanant des établissements d'enseignement et de recherche français ou étrangers, des laboratoires publics ou privés. 


\title{
Towards the Automatic Tuning of Linear Controllers Using Iterative Learning Control Under Repeating Disturbances
}

\author{
Oktay Koçan ${ }^{1}$, Charles Poussot-Vassal ${ }^{1}$ and Augustin Manecy ${ }^{1}$
}

\begin{abstract}
In this paper, iterative learning control (ILC) is proposed as an alternative approach that can be used to simplify the design of linear controllers for rejecting repetitive disturbances affecting a system. Since ILC is a data-driven method, its usage allows rejecting repetitive disturbances without a priori knowledge of their nature. The benefits of ILC appear especially when a system is subject to complex repetitive disturbances. This is simply because it would require a control designer to spend much more efforts to obtain similar results applying a state augmentation by building models for the same disturbances (Internal-Model-Principle based control). Accordingly, this paper first shows the equivalence of performing state augmentation and applying ILC in case of a simple sinusoidal repetitive disturbance. Next, a workflow named Learning Based Controller Tuning ( $L B C T)$ is proposed to simplify the parameter tuning of linear controllers under repetitive disturbances. The feasibility of LBCT is analysed by testing the ILC on a system subject to complex repetitive disturbances in two different forms: linearly combined sinusoidal signals and non-linearly combined sinusoidal signals. The results demonstrate that ILC can successfully learn the required controller parameters to reach a good rejection performance against the repetitive disturbances without a need of modelling them. This supports the fact that the tuning of linear controllers may be automated by integrating an ILC to the system.
\end{abstract}

\section{INTRODUCTION}

In real-life applications, the control performance is an important issue. The performance of a controller is directly related to its capability of dealing with uncertainties and disturbances that the system is subject to. The main source of system uncertainties can be related to the modelling of the dynamics. The dynamics of most real-life systems are usually governed by a set of nonlinear equations whose modelling depends on the understanding of the system's behaviour by the designer. Consequently, it is a common fact to have some residual uncertainty due to neglected or non-modelled dynamics. Moreover, it is also possible for the real system's dynamics to gradually deviate from the proposed model as a result of some external effects during the operation process (e.g. plant aging) such that the model starts to possess an increased uncertainty over time. Besides the uncertainties related to system modelling, another important control problem is to deal with the external disturbances at the system output. These disturbance issues have been addressed so far by considering two different approaches. One can either design a controller that is robust to some bounded disturbance or diminish the effect of the disturbance by using the knowledge of the disturbance in the controller after measuring or estimating it [1].

\footnotetext{
${ }^{1}$ Onera - The French Aerospace Lab, F-31055 Toulouse, France, <firstname. lastname>donera.fr
}

The state feedback control is an intuitive and a well established method for controlling dynamical systems. One can find it quite natural to build controllers based on the states of a dynamical system since the state variables can be used to anticipate the future behaviour of the system [2]. Accordingly, it is not a suprise that the state feedback method has been referred to since 70 s when analysing the disturbance rejection problems [3]. Although there are many more advanced robust methods developed to handle system disturbances such as Sliding Mode Control (SMC) [4], Active Disturbance Rejection Control (ADRC) [5] or Input Load Disturbance Rejection (ILDR) [6], the simplest one among all can be seen as the pole (or eigenvalue) placement method that uses state feedback through a linear controller structure. Furthermore, as a common practice one should include the disturbance dynamics in the controller tuning or the controller structure [7]. In order to lessen the effect of disturbance on the system, one can simply augment the state-space of the nominal system with the model of the disturbance and then apply the pole placement method. This type of approach becomes quite useful when dealing with periodic disturbances for which the structure is known. Some examples to these type of disturbances can be seen as a step signal, a sinusoidal signal at some specific frequency or a combination of several sinusoidal signals with different frequencies. Since the dynamics of such disturbances has periodic behaviours, they have a known structure and can be eliminated if their structure is utilised in the design of the controller.

Apart from traditional approaches such as state feedback control, it is also possible to adress the problem of periodic disturbances with a completely different perspective called the Iterative Learning Control (ILC) which can be seen as a specific version of the Repetitive Control ( $R C$ ). ILC is a data-driven method that is based on feedforward update by means of error filtering. It enables vanishing the repetitive errors that occur between the system runs (iterations) and it is traditionally used to "optimise" the tracking performance of repetitive trajectories for over a decade. One can obtain high trajectory tracking performance for motion control systems via feedforward control and in the case of a system that repeats the same task, the ILC can be used as the feedforward method to increase the system performance [8]. It should be noted that the ILC performance is highly dependent on the type of the system disturbance. Since ILC is based on remembering the errors from the previous runs and compensating for the repeating errors, it becomes a powerful approach when dealing with repetitive disturbances. 
Accordingly, the ILC performance can significantly reduce when the system is subject to non-repetitive disturbances simply because ILC will also remember the non-repeating error from the previous run. Due to this drawback, ILC is usually applied on a pre-designed closed-loop system which compensates for the non-repetitive characteristics of the system. Despite this fact, one can still think of many concepts affected by repeating disturbances where ILC can be applied such as fluid dynamics, helicopters, circuits etc.

If one considers a closed-loop system with a state feedback controller and an output repetitive disturbance, it would be quite natural to expect a similarity between the disturbance rejections by the state augmentation and the ILC integration. In this paper, we propose that one can use the ILC approach to simplify the design of a traditional state feedback controller for the systems that are subject to repetitive disturbances with complex forms. In similar words, ILC may be an easier solution for finding the proper parameters of a state feedback controller that will attenuate an existing repetitive disturbance since it requires no modelling of the disturbance and does not get affected by the complexity of the structure of the repetitive disturbance. Hence, the analysis provided in this paper is based on a comparison of both methods.

The work in this paper hereafter was given in the following order: in Sec. II, we explain the traditional state feedback approach for disturbance rejection and show its relation to ILC; in Sec. III, we give insight to the ILC approach and compare it to the an augmented state feedback controller; In Sec. IV we introduce our LBCT workflow; in Sec. V, we analyse the feasibility of this workflow; in Sec.VI, we give some conclusions.

\section{ILLUSTRATION FOR DISTURBANCE REJECTION}

\section{A. Model and disturbance}

Let us consider a linear time invariant dynamical system described by a second order continuous transfer function. The state-space form of the system is given as:

$$
\begin{aligned}
\dot{x}(t) & =A x(t)+B u(t), \\
y(t) & =C x(t)+d(t) .
\end{aligned}
$$

where $x(t), y(t) \in \mathbb{R}^{2}, u(t) \in \mathbb{R}^{1}$ and

$$
A=\left[\begin{array}{cc}
-3.5014 & -3.0003 \\
1 & 0
\end{array}\right], B=\left[\begin{array}{l}
0 \\
1
\end{array}\right], C=\left[\begin{array}{ll}
1 & 0 \\
0 & 1
\end{array}\right] .
$$

Similarly, we consider the repetitive disturbance to be in the sinusoidal form, i.e $d(t)=a \cdot \sin (w t)$ where $a$ and $w$ are the amplitude and the pulsation of the signal, respectively.

\section{B. Traditional state feedback approach}

In order to be able to reject such a repetitive disturbance $d(t)$, one should augment the state-space of the system with the disturbance model. Therefore, we define an output $\tilde{z}(s)$ by considering the Laplace transform of $d(t)$ with the system reference $r(t)$ and the system output $y(t)$ :

$$
\tilde{z}(s)=\frac{a w}{s^{2}+w^{2}}(\tilde{r}(s)-\tilde{y}(s)),
$$

for which the state-space is written as:

$$
\left[\begin{array}{c}
\ddot{z}(t) \\
\dot{z}(t)
\end{array}\right]=\left[\begin{array}{cc}
0 & -w^{2} \\
1 & 0
\end{array}\right]\left[\begin{array}{c}
\dot{z}(t) \\
z(t)
\end{array}\right]+\left[\begin{array}{c}
a w \\
0
\end{array}\right] \epsilon(t)
$$

where $\epsilon(t)=r(t)-y(t)$ is the error. Next, one can use (4) to augment the system given by (1) and (2) leading to (5). Assuming that we only focus on the second output at which the disturbance occurs, i.e $C_{2}=\left[\begin{array}{ll}0 & 1\end{array}\right]$, the augmented system is built as follows:

$$
\left[\begin{array}{c}
\dot{x} \\
\ddot{z} \\
\dot{z}
\end{array}\right]=\left[\begin{array}{ccc}
A & 0_{2 \times 1} & 0_{2 \times 1} \\
-C_{2} w & 0 & -w^{2} \\
0_{1 \times 2} & 1 & 0
\end{array}\right]\left[\begin{array}{c}
x \\
\dot{z} \\
z
\end{array}\right]+\left[\begin{array}{c}
B \\
0 \\
0
\end{array}\right] u+\left[\begin{array}{c}
0 \\
a w \\
0
\end{array}\right] r .
$$

At this point, one may classically apply the pole placement method with $u=-K x_{a u g}$ to move the disturbed system's poles to the desired locations that will produce the required tracking and disturbance rejection $\left(x_{a u g}=\left[\begin{array}{lll}x & \dot{z} & z\end{array}\right]^{T}\right.$ is the augmented state vector). In order to see the efficiency of this method in terms of repetitive disturbance rejection, a numerical simulation is carried out with $r=0, a=0.2$ and $w=1 \mathrm{rad} / \mathrm{s}$ which are the reference input, the disturbance amplitude and the disturbance frequency, respectively. Furthermore, the new pole locations are chosen to be the ones given by Bessel poles for a 4th order system which produce a settling time of $1 \mathrm{sec}$ (i.e. $p_{1,2}=-4.016 \pm 5.072 i$ and $p_{3,4}=-5.528 \pm 1.655 i$ ). The model used for simulation is composed of an inner loop (state feedback) and an outer loop (augmented state feedback). The structure of this model can be seen in Fig. 1. One can observe that it serves both for simulating the augmented system and the ILC system by means of a switch. The curves denoted as 'ASFB' in Fig. 2 show the simulation results with the ILC switched-off (i.e. ' $S .1$ ' in Fig. 1). It can be seen that the augmented system is capable of perfectly rejecting the sinusoidal disturbance in about 1.5 seconds.

\section{Iterative Learning Control based approach}

We suggest that integrating an ILC into an unaugmented state feedback system would produce a similar behaviour to the one of the augmented state feedback system in terms of the repetitive disturbance rejection. This is due to the fact that ILC has an integral action as a result of its update equation (7). The following section provides details for the ILC algorithm utilised in our approach and the corresponding disturbance rejection results are demonstrated in Fig. 2.

\section{ILC APPROACH FOR DISTURBANCE REJECTION}

\section{A. Algorithm overview}

The ILC technique we apply in this work belongs to the norm-optimal ILC (NO-ILC) class and it is based on the minimization of a quadratic cost function $(J)$ using Lagrange multipliers. Even though other selections for $J$ are also possible considering different requirements, we define $J$ for our case as below:

$$
\begin{aligned}
J\left(u_{i+1}\right) & =e_{i+1}^{T} W_{e} e_{i+1}+u_{i+1}^{T} W_{u} u_{i+1} \\
& +\lambda\left[\left(u_{i+1}-u_{i}\right)^{T}\left(u_{i+1}-u_{i}\right)\right]
\end{aligned}
$$




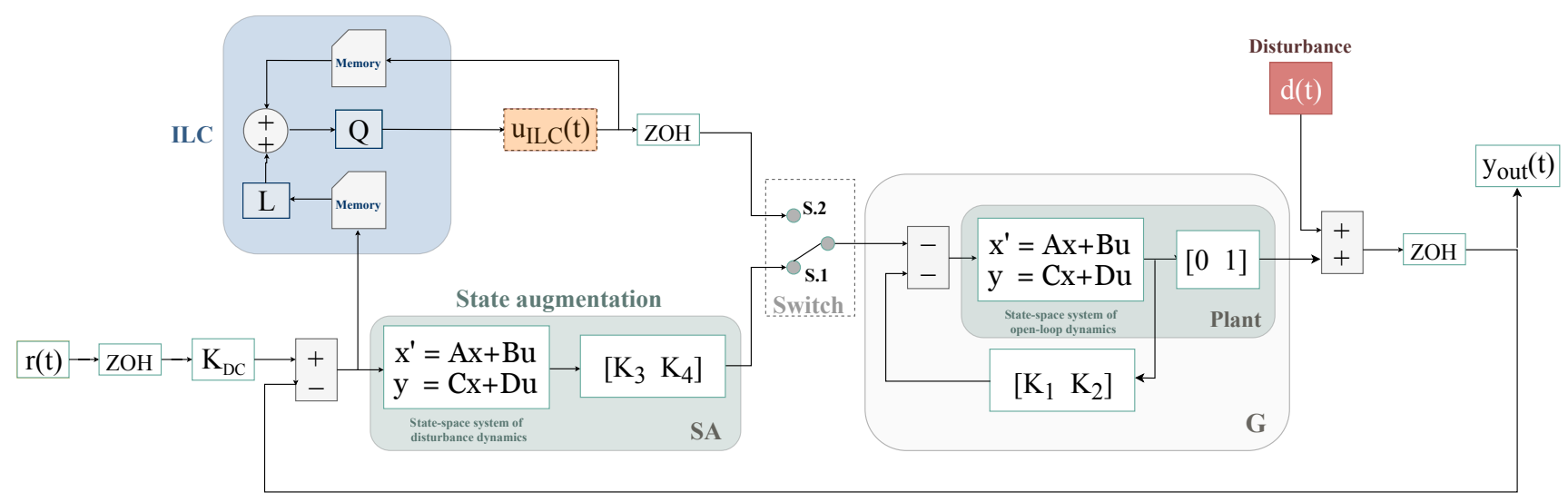

Fig. 1: Simulation model

where $i$ is the iteration index of ILC; $e_{i+1} \in \mathbb{R}^{N}$ is the system's current tracking error; $u_{i+1} \in \mathbb{R}^{N}$ is the system's current input; $u_{i} \in \mathbb{R}^{N}$ is the system's previous input; $\lambda \in$ $\mathbb{R}^{1}$ is the Lagrange multiplier; $W_{e}=\rho I \in \mathbb{R}^{N \times N}$ and $W_{u}=$ $I \in \mathbb{R}^{N \times N}$ are the respective weighting matrices where $I$ is the identity matrix. Consider that $N$ is a variable depending on the simulation time that determines the size for the given vectors and matrices.

The NO-ILC used hereafter was adapted from [9]. In brief, the followed procedure develops through finding the optimizing ILC filters via analytically solving a cost function. The cost function (6) is written by considering the system's current output tracking error $\left(e_{i+1}\right)$, the current input $\left(u_{i+1}\right)$ and the input difference $\left(u_{i+1}-u_{i}\right)$ to avoid chattering. Then, the optimization is done via the method of Lagrange multipliers. Furthermore, the convergence of the ILC algorithm is determined by the tuning of two scalar parameters: $\rho>0$ and $\lambda>0$ which are the input weight and the Lagrange multiplier, respectively. The tuning of these parameters is a heuristic process done under the convergence conditions of $\|Q\|_{2}<1$ and $\|Q L\|_{2} \leq 0.5 / \sqrt{\rho+\lambda}$. As in $L Q$-control, from the performance viewpoint, there exists a trade-off between $\rho$ and $\lambda$ (see [9] for details).

The ILC update equation (7) has two filters named learning filter $\left(L \in \mathbb{R}^{N \times N}\right)$ and $Q$-filter $\left(Q \in \mathbb{R}^{N \times N}\right)$ given by (8) and (9), respectively.

$$
\begin{aligned}
u_{i+1} & =Q u_{i}+Q L e_{i}, \\
Q & =\left((\lambda+\rho) I+G^{T} G\right)^{-1}\left(\lambda I+G^{T} G\right), \\
L & =\left(\lambda I+G^{T} G\right)^{-1} G^{T},
\end{aligned}
$$

where $G$ is the lifted-matrix (see [10]) of the internal system in Fig. 1.

\section{B. Simulation example}

The same model in Fig. 1 is used for the ILC simulation. However, this time the augmented state feedback loop in the model is switched off such that the system is only composed of the ILC input and the inner state feedback system for which the gain values stay the same as in the augmented state feedback system (i.e. 'S.2' is 'ON' in Fig. 1). The ILC input, $u_{I L C}$ is calculated off-line and updated after each run of the model. The disturbance parameters remain the same as before $(a=0.2, w=1 \mathrm{rad} / \mathrm{s})$ and the initialization of the ILC is performed with the parameters shown on Table I where $\rho, \lambda$ and $M$ are selected by trial-and-error.

TABLE I. NO-ILC initialisation

\begin{tabular}{|l|c|}
\hline Sample time, $T_{s}$ & $0.01 \mathrm{sec}$. \\
Simulation time, $T_{\text {sim }}$ & $50 \mathrm{sec}$. \\
Initial states, $x$ & {$\left[\begin{array}{ll}0 & 0\end{array}\right]^{T} \in \mathbb{R}^{2 \times 1}$} \\
Initial ILC input, $u_{I L C}$ & 0 \\
Number of ILC iterations, $M$ & 1000 \\
Weight on the error, $W_{e}$ & $\rho I \in \mathbb{R}^{N \times N}$ \\
Weight on the system input, $W_{e}$ & $I \in \mathbb{R}^{N \times N}$ \\
$\rho$ & 0.001 \\
$\lambda$ & 0.1 \\
\hline
\end{tabular}

It is expected from ILC to learn the control signal of the augmented state feedback system as the iterations continue. Theoretically, such an expectation is rather normal since for a linear system there exists only one single solution. Fig. 2 compares the control and output signals of the augmented state feedback system to the ones of ILC at $1000^{t h}$ iteration. It can clearly be observed that both methods successfully reject the given disturbance. The only difference, however, is that ILC is capable of handling the transients that are inevitable for the augmented state feedback system at the beginning of the simulation. This is simply because of the fact that ILC can detect the repeating error patterns between system runs and anticipate for them. Furthermore, the augmented state feedback system can completely reject the disturbance making the output exactly zero while the system with ILC still has some remaining oscillations in its output whose magnitude is less than 0.00074 ( $\% 0.37$ of the original disturbed output). Yet, these oscillations can be considered as insignificant.

\section{Learning Based Controller Tuning (LBCT)}

The simulation results so far has shown that the ILC can interchangeably be used with the traditional state feedback in case of a single sinusoidal repetitive disturbance. Although one can argue about the necessity of replacing a well understood traditional method with a data-driven one, the benefits of ILC arise when the complexity of the disturbance increases. Since the state augmentation method is grounded on the exact knowledge of disturbance model, it requires 

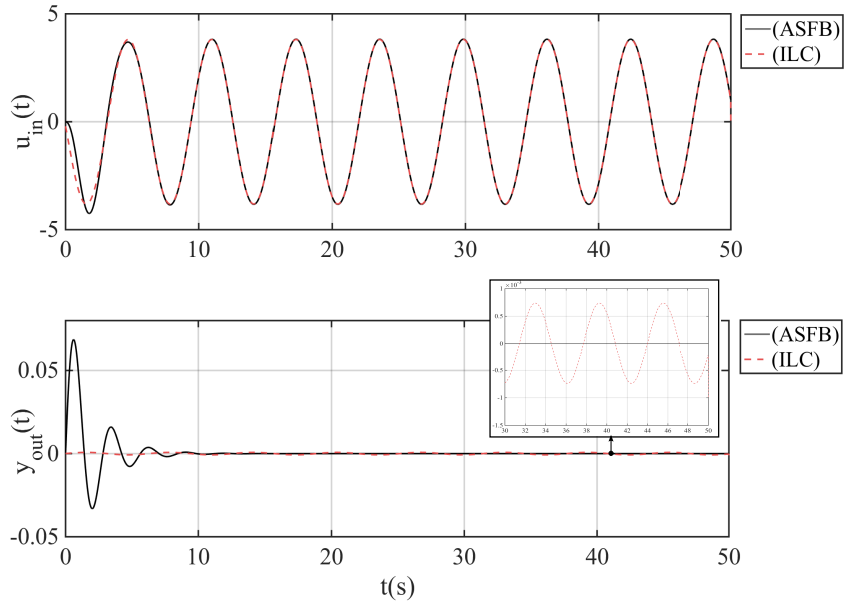

Fig. 2: Augmented state feedback system vs. ILC system

carefully acquiring all the frequencies inside the disturbance signal for proper attenuation. Accordingly, the designer's work gets more complicated and is open to errors as the number of frequencies in the disturbance signal becomes larger. In order to easily deal with complex repetitive disturbances without needing to build their models, we propose a Learning Based Controller Tuning (LBCT) procedure that combines ILC with model identification (see Workflow 1).

Workflow 1 first uses the ILC to find an optimal system input $u_{i=M}^{*}(t)$ that rejects a repeating disturbance $d(t)$ (Steps 1-3). Next, it applies FFT to obtain the corresponding frequency data set $\left\{\omega_{j}, \phi_{j}\right\}_{j=1}^{N_{f}}$ where $j \in \mathbb{N}^{+}, \omega_{j} \in \mathbb{R}^{+}$, $\phi_{j} \in \mathbb{C}^{1 \times 1}$ and $N_{f} \in \mathbb{N}^{+}$are the frequency data index, the frequency at $j$, the frequency response at $\omega_{j}$ and the number of frequency samples, respectively (Step 4). Then, the workflow includes a Loewner framework ( [11] and [12]) based method to make an approximate fit on this data with a rational function $H(s) \in \mathcal{H}_{\infty}$ (Step 5). Finally, it is proposed that the properties of the transfer function $H(s)$ can be used for designing linear controllers via state feedback, loop shaping etc. (Step 6).

\section{LBCT UNDER COMPLEX DisTURBANCES}

Let us now assess the feasibility of LBCT approach by showing that the ILC is capable of detecting all the repeating frequencies for two different complex repetitive disturbances. Thus, we first consider a linear combination of repetitive disturbance signals and second extend the analysis to a non-linear combination of repetitive disturbance signals. However, we limit our analysis in this work to a curve fitting application on the frequency data of the ILC. Hence, we leave the process of obtaining the corresponding linear controller transfer functions for a future work related to a real life problem.

\section{A. Linearly combined disturbance signals}

The ILC's ability of rejecting more complex disturbances can be tested by considering a repetitive disturbance that is a linear combination of three sinusoidal signals with different frequencies but same amplitudes. Using the same form given in Section II for the sinusoidal signals, one can write the corresponding complex disturbance $d(t)$ as:

$$
a\left(\sin \left(w_{1} t\right)+\sin \left(w_{2} t\right)+\sin \left(w_{3} t\right)+\sin \left(w_{4} t\right)\right)
$$

where $a=0.2, w_{1}=0.25 \mathrm{rad} / \mathrm{s} \approx 0.04 \mathrm{~Hz}, w_{2}=$ $0.5 \mathrm{rad} / \mathrm{s} \approx 0.08 \mathrm{~Hz}, w_{3}=0.75 \mathrm{rad} / \mathrm{s} \approx 0.12 \mathrm{~Hz}$ and $w_{4}=1 \mathrm{rad} / \mathrm{s} \approx 0.16 \mathrm{~Hz}$. One can view this signal in Fig. 3. Furthermore, the desired reference signal of the system which is also the initial ILC signal was set to zero and the initialisation of the ILC algorithm was done under the same simulation parameters previously given on Table I. The simulation results are given in Fig. 4 and 5 which show the variation of system inputs and the corresponding system outputs in the iteration axis, respectively. The system inputs in Fig. 4 can be seen as the ILC signals for the corresponding iterations since each new ILC signal was defined as the new system input. It can clearly be seen from the results in Fig. 5 that the ILC algorithm was able to gradually attenuate the disturbance effect to a huge degree such that the magnitude of the output at the last iteration reached below 0.00242 . Moreover, one can observe in Fig. 4 how the ILC signal tended towards a signal which is the close approximation of the inverse of the disturbed system.

It is also possible to portray the efficiency of the ILC by analysing the frequency components $\left\{\omega_{j}, \phi_{j}\right\}_{j=1}^{N_{f}}$ of the last ILC signal. For this purpose, we estimate the frequency response of the ILC system at last iteration supposing an impulse signal as its input and the last ILC signal as its output. Then, we use a method based on the Loewner framework detailed in [11] and [12] to apply an approximate fit on the frequency data with a rational function $H(s)$. The resulting fit and the frequency response data of the ILC system can be reviewed in Fig. 6. Expectedly, the ILC system has peaks nearly at the frequencies of the disturbance (10) which proves its capability of singling out each repetitive component in the disturbance.

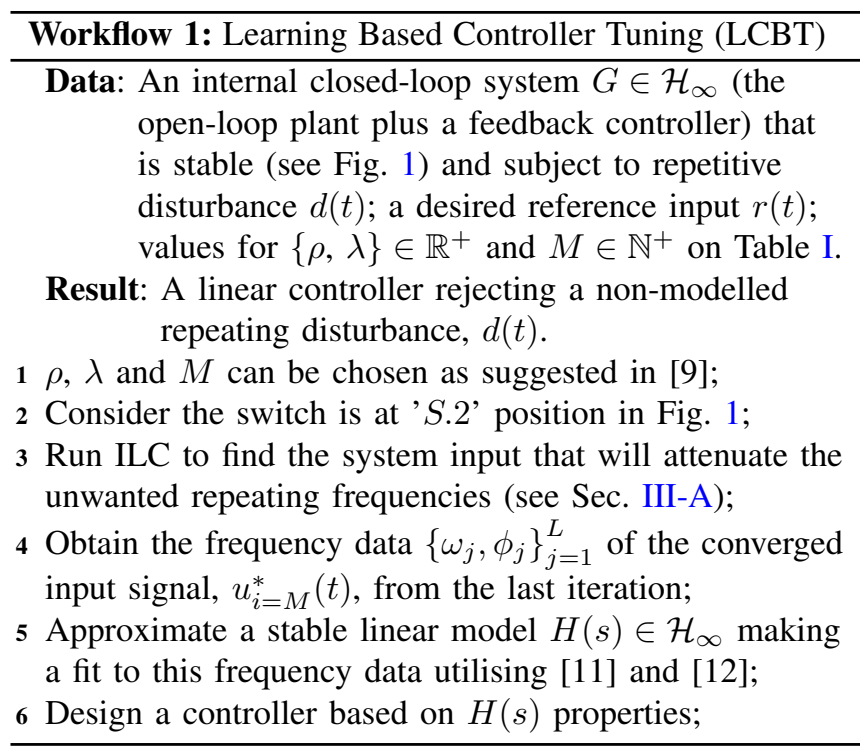

\section{B. Nonlinearly combined disturbance signals}

After observing a satisfactory attenuation performance against three linearly combined repetitive sinusoidal disturbance signals, we extend our analysis this time assuming that 


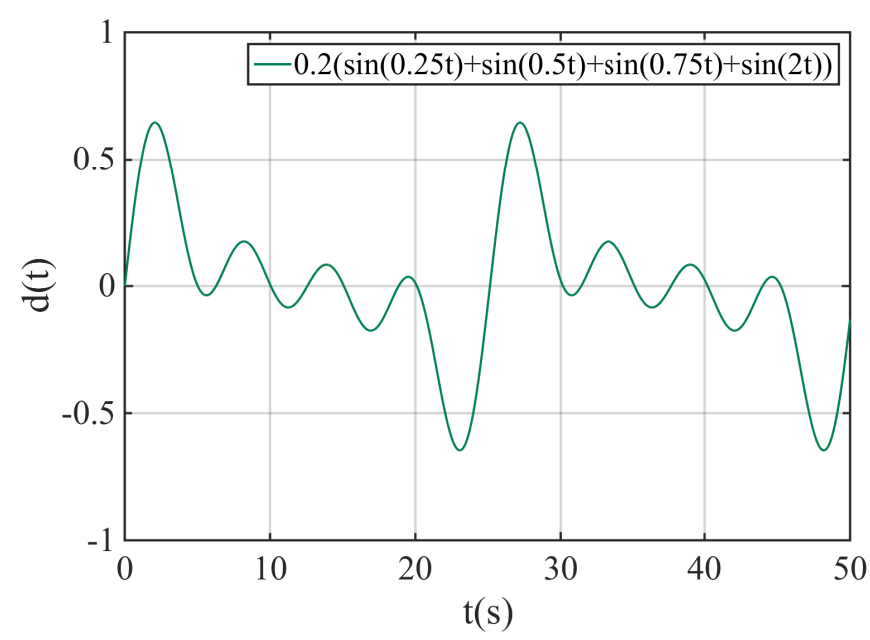

Fig. 3: Linearly combined disturbance signal

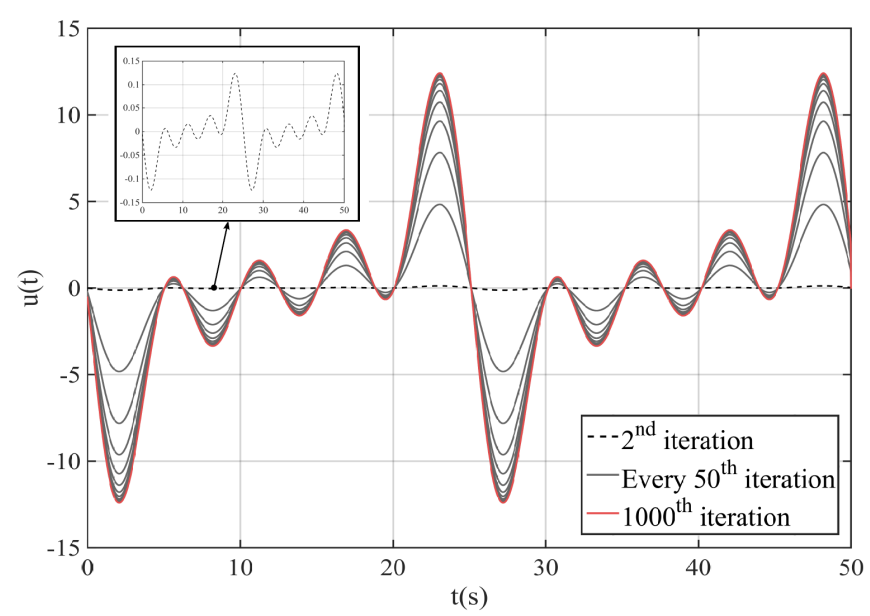

Fig. 4: System input along iterations

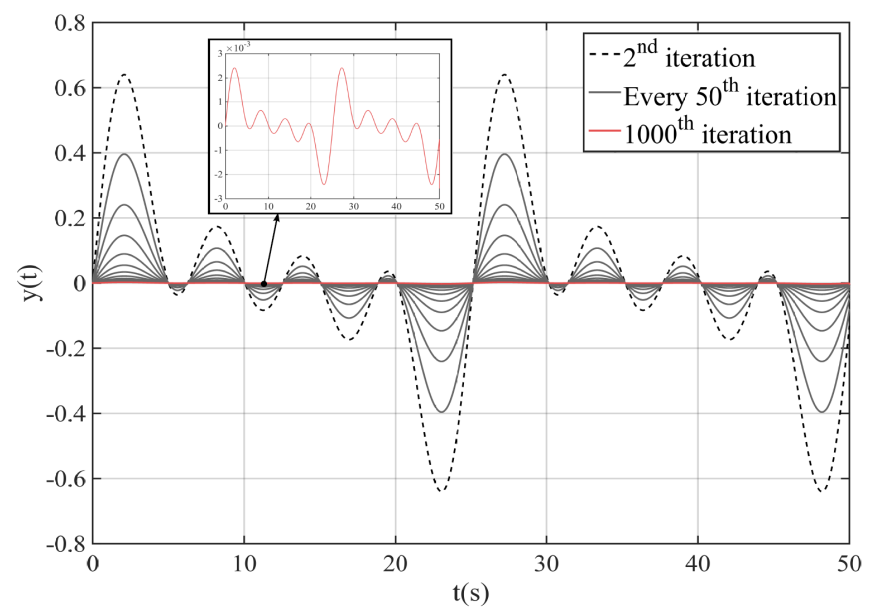

Fig. 5: System output along iterations

the disturbance signal is a non-linear combination of three repetitive sinusoidal signals with different frequencies. Thus, the disturbance $d(t)$ is considered as follows:

$$
\begin{aligned}
& 0.25\left(\left(0.7\left(0.15-0.8 \sin \left(w_{1} t\right)\right)^{2}-0.6 \sin \left(w_{2} t\right)\right)^{3} \ldots\right. \\
& \left.\quad \ldots-0.35\left(\sin \left(w_{3} t\right)\right)^{2}\right)
\end{aligned}
$$

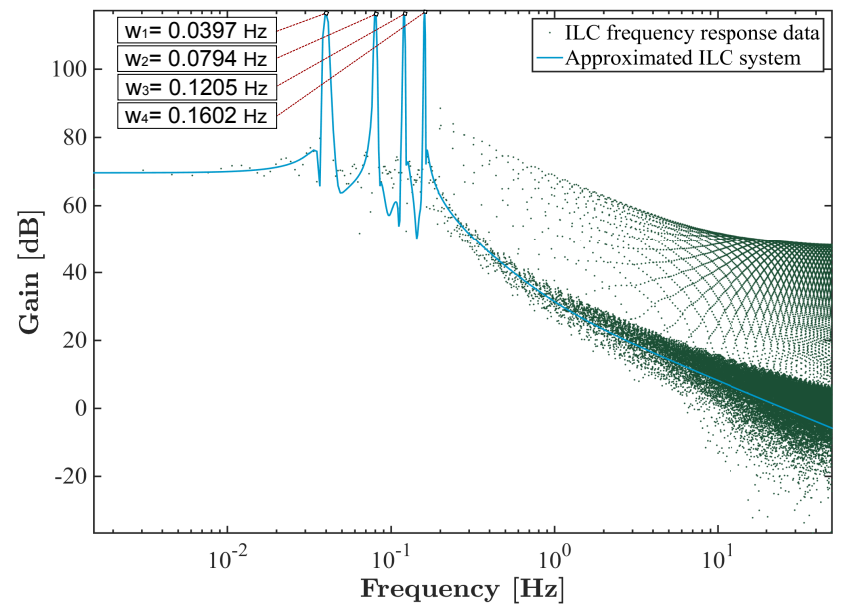

Fig. 6: Frequency response of the ILC system and its approximated model, $H(s)$

where $w_{1}=0.25 \mathrm{rad} / \mathrm{s} \approx 0.04 \mathrm{~Hz}, w_{2}=0.75 \mathrm{rad} / \mathrm{s} \approx$ $0.12 \mathrm{~Hz}$ and $w_{3}=1 \mathrm{rad} / \mathrm{s} \approx 0.16 \mathrm{~Hz}$. Again, the ILC was initialised using Table I and the desired system reference was set to zero. It should be noted that the ILC algorithm used here is the same as the one of the previous analysis and the only difference comes from the disturbance signal. Accordingly, the ILC can be seen as a model free approach from the point of disturbance rejection since the ILC filters (i.e. (8) and (9)) use only the information of the existing closed-loop system $G$. This type of data-based approach converts a difficult modelling problem to a simple filtering operation.
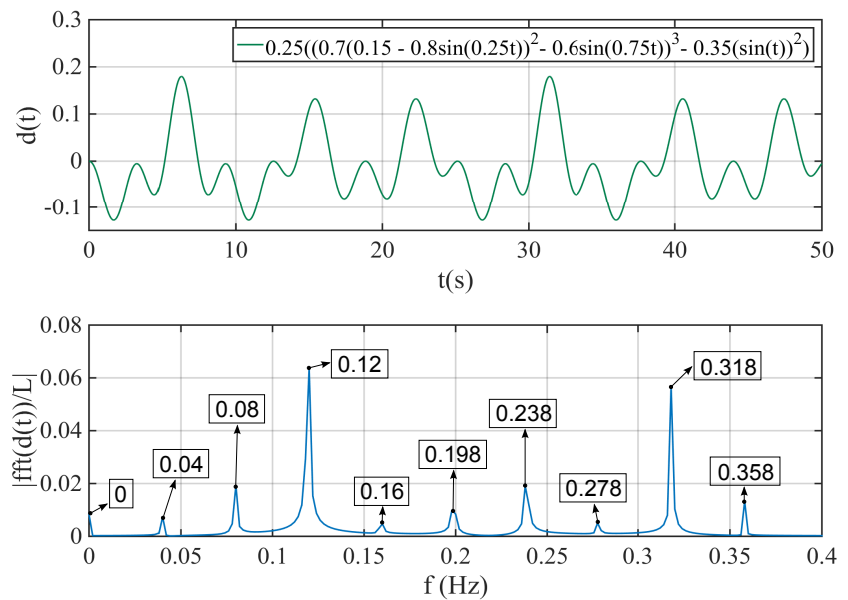

Fig. 7: (Top) Non-linearly combined disturbance signal; (Bottom) Fast Fourier transform of the disturbance signal showing the main frequencies ( $L$ : frequency data length).

The disturbance signal (11) and its frequency components are depicted in Fig. 7. The results from the ILC analysis are provided in Fig. 8 and Fig. 9 which show the evolutions of the system inputs and outputs, respectively. One can notice that the system output converges towards the desired reference as the ILC gradually calculates the proper inverse of the disturbed system. Moreover, analogically to the previous subsection, a frequency response data analysis was done for the converged ILC system and a model was obtained again by applying an approximated fit on the corresponding data 
following [11] and [12]. The result was given in Fig. 10 where one can observe nearly the same frequencies as those found in the disturbance signal in Fig. 7. Hence, we can clearly conclude that the ILC algorithm successfully detects the disturbance frequencies to be attenuated in order to reach the desired reference and it can be used for augmented state feedback control tuning.

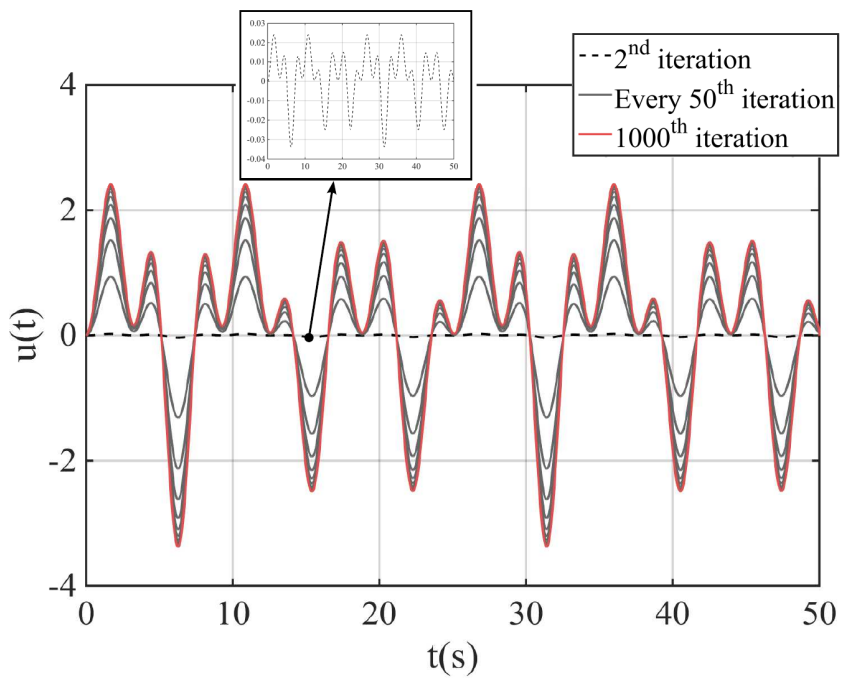

Fig. 8: System input along iterations

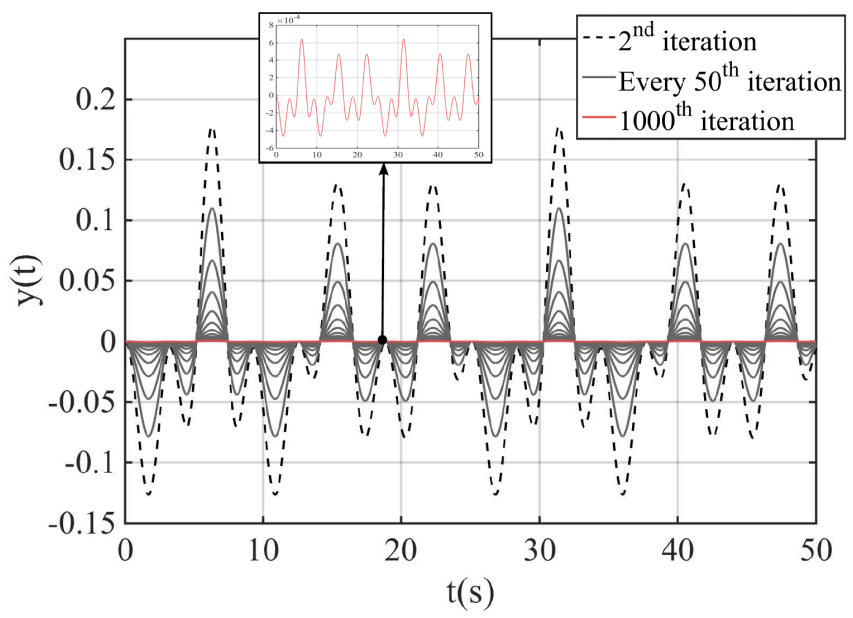

Fig. 9: System output along iterations

\section{CONCLUSION}

The augmented state feedback control and ILC were found to be equivalent in rejecting a simple sinusoidal repetitive disturbance. According to the results, the ILC demonstrated a rather successful rejection performance and it can be considered as superior to state feedback control since it was able to gradually remove the repeating errors and anticipate for the transients in the response. Hence, instead of trying to correctly model the repetitive disturbances, one can utilise ILC to learn the required system inputs that will reject the existing repetitive disturbances. Then, these learned signals can be utilised for automatically tuning the parameters of linear controllers.

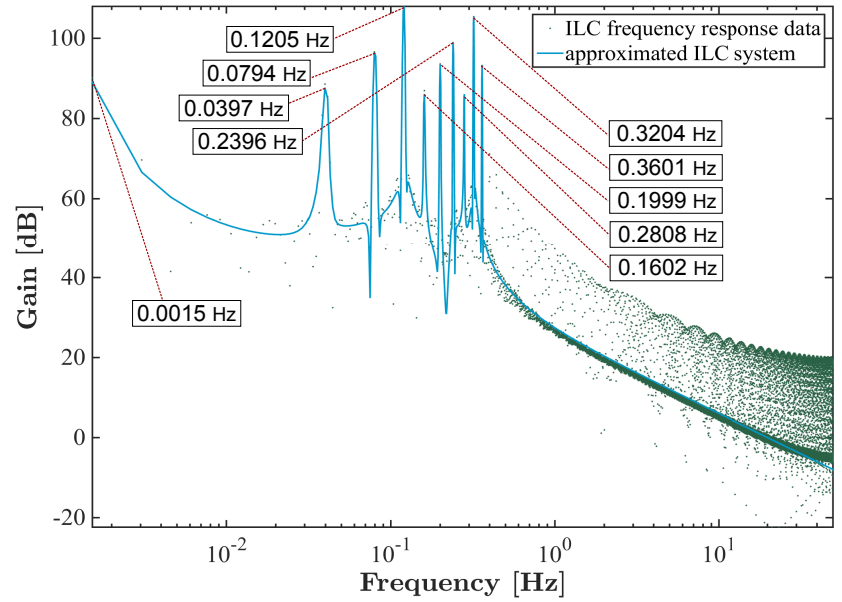

Fig. 10: Frequency response of the ILC system and its approximated model, $H(s)$

Our LBCT workflow puts the presented ideas in compact form and as a future work, we consider implementing this tuning approach on a real life problem.

\section{REFERENCES}

[1] R. Sanz, P. Garcia, and P. Albertos, "Active disturbance rejection by state feedback: Experimental validation in a 3-dof quadrotor platform," in 54th Annual Conference of the Society of Instrument and Control Engineers of Japan (SICE), no. 15491066. Hangzhou, China: IEEE, July 2015, pp. 1127-1131.

[2] K. Astrom and R. Murray, Feedback Systems: An Introduction for Scientists and Engineers. New Jersey: Princeton University Press, 2008.

[3] F. Blanchini and M. Sznaier, "Persistent disturbance rejection via static state feedback," in Proceedings of 1994 33rd IEEE Conference on Decision and Control, no. 5032337, Lake Buena Vista, FL, USA, USA, December 1994, pp. 3159-3164.

[4] B.-Z. Guo and F.-F. Jin, "The active disturbance rejection and sliding mode control approach to the stabilization of the euler-bernoulli beam equation with boundary input disturbance," Automatica, vol. 49, no. 9, pp. 2911-2918, 2013.

[5] H. Feng and B.-Z. Guo, "Active disturbance rejection control: Old and new results," Annual Reviews in Control, 2017.

[6] W. Zhang, L. Xia, and C. Wang, "Optimal input load disturbance rejection controller design for typical integrating processes based on imc structure," in Real-time Computing and Robotics (RCAR), IEEE International Conference on. IEEE, 2016, pp. 656-661.

[7] M. Steinbuch and R. Molengraft, "Iterative learning control of industrial motion systems," in IFAC Proceedings Volumes, ser. 26, vol. 33. Darmstadt, Germany: Elsevier, September 2000, pp. 899-904.

[8] S. Yu, "Enhanced iterative learning control with applications to a wafer scanner system," phdthesis, University of California, Berkeley, 2011. [Online]. Available: https://escholarship.org/uc/item/6bc4x5k8\#main

[9] M. Norrlof, "Iterative learning control: Analysis, design, and experiments," Ph.D. dissertation, University of Linkoping, 2000. [Online]. Available: http://users.isy.liu.se/rt/mino/pub/thesis653.pdf

[10] D. Bristow, M. Tharayil, and A. Alleyne, "A survey of iterative learning control: A learning-based method for high-performance tracking control," IEEE Control Systems Magazine, vol. 26, no. 3, pp. 96-114, Mar. 2006.

[11] A. Antoulas, S. Lefteriu, and A. Ionita, Model reduction and approximation theory and algorithms, ser. SIAM, Philadelphia. P. Benner, A. Cohen, M. Ohlberger and K. Willcox Eds, 2016, ch. A tutorial introduction to the Loewner framework for model reduction.

[12] A. J. Mayo and A. C. Antoulas, "A framework for the solution of the generalized realization problem," Linear Algebra and its Applications, vol. 425, no. 2, pp. 634-662, 2007. 